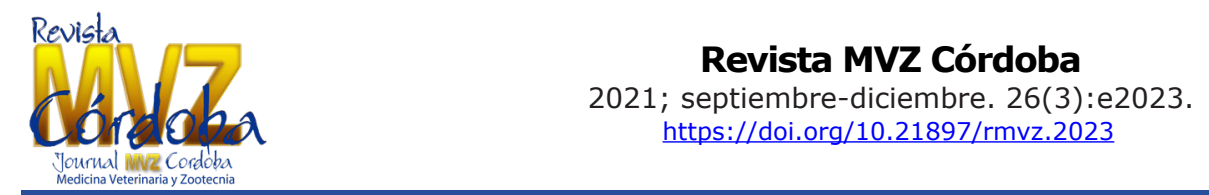

\title{
La administración repetida de vacuna autógena disminuye la papilomatosis en hembras bovinas
}

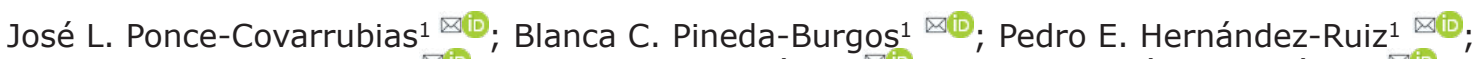 \\ Edgar Valencia-Franco ${ }^{凶(\mathbb{D}} ;$; Ricardo Vicente-Pérez ${ }^{凶}$; Ethel C. García y González ${ }^{1 *} \bowtie(\mathbb{D}$
}

\begin{abstract}
${ }^{1}$ Universidad Autónoma de Guerrero (UAGro), Escuela Superior de Medicina Veterinaria y Zootecnia No. 3, Tecpan de Galeana, Guerrero, México. Benemérita Universidad Autónoma de Puebla, Facultad de Ciencias Agrícolas y Pecuarias, Tlatlauquitepec, Puebla, México. 3universidad de Guadalajara, Centro Universitario de la Costa Sur, Autlán de Navarro, Jalisco, México.

${ }^{*}$ Correspondencia: eth cat@hotmail.com
\end{abstract}

Recibido: Mayo 2020; Aceptado: Mayo 2021; Publicado: Junio 2021.

\section{RESUMEN}

Objetivo. El presente estudio tuvo como objetivo evaluar el control de la papilomatosis bovina (PB) con vacuna autógena en condiciones del trópico de Guerrero. Materiales y métodos. En el experimento se usaron 18 hembras bovinas que fueron divididas en tres tratamientos de acuerdo al rancho (6 hembras/grupo). Tratamiento 1 (T1-inmunovacuna), tratamiento 2 (T2-histovacuna) y tratamiento 3 (T3-plasmaterapia). Resultados. Al inicio del estudio se encontró una alta incidencia de papilomas en las hembras bovinas [(T1 (190 papilomas), T2 (95 papilomas) y T3 (100 papilomas)]. Con la administración de la vacuna autógena disminuyó significativamente (T1 y T3) el número de papilomas a través de las aplicaciones de la 1 (147 y 66 papilomas) a la 5 (10 y 9 papilomas); $(p<0.05)$. También existió una diferencia significativa entre tratamientos de acuerdo al periodo de aplicación de la vacuna ( 3 a la 5 ) disminuyendo el número de papilomas (efecto periodo; $\mathrm{p}<0.05$ ). Por su parte, se observó una interacción tratamiento $x$ periodo disminuyendo el número de papilomas en la cuarta y quinta administración (T1 y T3; p<0.05). Conclusiones. La administración repetida de la vacuna autógena en las hembras bovinas disminuyó el número de papilomas entre la cuarta y quinta aplicación en los tratamientos 1 y 3.

Palabras clave: Autovacuna; carcinoma; daño en la piel; tratamiento; verrugas (Fuente: $M e S H$ ).

\section{ABSTRACT}

Objective. The present study aimed to evaluate the control of bovine papillomatosis (BP) with autogenous vaccine under the tropical conditions of Guerrero State. Materials and methods. In the experiment 18 bovine females were used, that were divided into three treatments according to the ranch (6 females / group). Treatment 1 (T1-immune vaccine), treatment 2 (T2-histovaccine) and treatment 3 (T3-plasma therapy). Results. At the beginning of the study, a high incidence of papillomas was found in bovine females [(T1 (190 papillomas), T2 (95 papillomas) and T3 (100 papillomas)]. With the administration of the autogenous vaccine, the number of papillomas decreased significantly ( $\mathrm{T} 1$ and T3) through the applications from 1 (147 and 66 papillomas) to 5 (10 and 9

Como citar (Vancouver).

Ponce-Covarrubias JL, Pineda-Burgos BC, Hernández-Ruiz PE, Valencia-Franco E, Vicente-Pérez R, García-y-González EC. La administración repetida de vacuna autógena disminuye la papilomatosis en hembras bovinas. Rev MVZ Córdoba. 2021; 26(3):e2023. https://doi.org/10.21897/rmvz.2023 
papillomas $) ;(p<0.05)$. There was also a significant difference between treatments according to the period of application of the vaccine ( 3 to 5 ), decreasing the number of papillomas (period effect; $\mathrm{p}<0.05)$. On the other hand, a treatment $x$ period interaction was observed, decreasing the number of papillomas in the fourth and fifth administration (T1 and T3; $p<0.05$ ). Conclusions. The repeated administration of the autogenous vaccine in bovine females decreased the number of papillomas between the fourth and fifth application in treatments 1 and 3.

Keywords: Autovaccine; carcinoma; skin damage; treatment; warts (Sourse: MeSH).

\section{INTRODUCCIÓN}

En México, la región tropical es la de mayor potencial para la producción de ganado bovino doble propósito (1); la cual contribuye fuertemente a la nutrición de las comunidades más pobres y aisladas del país (2). Entre los principales estados productores de ganado bovino encontramos al estado de Guerrero, con 1.300 .000 cabezas, distribuidas en 42,000 unidades de producción. Este ganado se encuentra distribuido en las regiones Tierra Caliente, Norte, Costa Chica, Costa Grande y en el resto del estado $(39,18,16,16$ y $11 \%$; respectivamente) (2).

En el municipio de Cuajinicuilapa, región Costa Chica, una problemática importante son las enfermedades que afectan al ganado bovino, entre las que se encuentran principalmente: la brucelosis, tuberculosis, pasteurelosis, leptospirosis, anaplasmosis, babesiosis, colibacilosis, salmonelosis y papilomatosis bovina (PB) (2). La PB es una enfermedad infectocontagiosa viral, caracterizada por la formación de tumores en la piel y mucosas; de carácter tumoral benigno y de naturaleza fibroepitelial $(3,4)$.

Se ha comprobado que los papilomas son causados por virus (ADN) de papiloma bovino de tipo del 1 al 6 (VPB1 al 6 ) $(5,6)$, que tienen componentes antigénicos comunes, pero no poseen una adecuada reactividad inmunológica cruzada (7). Los virus tipo 1 y 2 afectan distintas regiones del cuerpo tal como cabeza, cuello, pene y mucosa vaginal; el tipo 3 solo piel; el tipo 4 al tracto digestivo; el tipo 5 y 6 afecta los pezones en forma de gránulos de arroz y en formas aplanadas, respectivamente $(3,4)$. Estas masas tumorales generalmente en la piel con aspecto de una coliflor con la superficie rugosa, o cubierta de costras, y de un color que varía del blanco al gris (8).
La PB afecta fuertemente la salud de los animales $y$, por ende, la economía de los productores. La enfermedad viral es común en los bovinos jóvenes, aunque producen poco daño y desaparecen espontáneamente (3). Los animales con lesiones a causa de las verrugas pueden sufrir infecciones bacterianas o parasitarias secundarias (miasis por vectores como insectos) (4). Por su parte, en las vacas con papilomas en los pezones y las ubres pueden dificultar el ordeño, asimismo causar mastitis (9). Cuando los fibropapilomas se localizan en las pezuñas en el espacio interdigital, cojinetes y talones, son dolorosos y pueden provocar desde cojeras hasta postración disminuyendo el consumo voluntario y la ganancia de peso (10). Lo anterior aunado al aspecto desagradable y el deterioro de las pieles representan una dificultad en la comercialización debido a que no pueden ser utilizadas en la industria de la peletería (3).

En este sentido, no es posible el establecimiento de normas de seguridad específicas para el control de la PB, debido a la naturaleza impredecible del virus. Sin embargo, se pueden tomar en cuenta algunas acciones para limitar el contagio: evitar el contacto directo entre animales (infectados - sanos), manejo de las instalaciones, manejo apropiado del material y equipo usados (11). Asimismo, debido a la presencia de ADN viral en fluidos corporales (sangre, leche, orina y semen) implementando programas de inseminación artificial, y evitando la transmisión vertical analizando bancos de semen (12).

Finalmente, se han sugerido múltiples tratamientos contra la PB, sin embargo, la efectividad reportada es altamente variada entre los tratamientos $(11.1 \%$ hasta $77.7 \%$, teniendo mayor efectividad los agentes químicos) (13). Intervención quirúrgica, vacuna contra el virus de la enfermedad de Newcastle, diaceturato de diazoaminodibenzamidina, inyección de preparados que contienen bismuto y antimonio, 
tratamientos homeopáticos con Thuja, ácido salicílico, látex de higuera, auto-hemoterapia y las vacunas autógenas preparadas con tejidos de verrugas del animal infectado $(14,15)$. Adicionalmente, han sido utilizados exitosamente en el tratamiento de la PB los fármacos estimulantes del sistema inmune como el levamisol y la ivermectina (16).

Por lo anteriormente mencionado, el objetivo del presente estudio fue evaluar el tratamiento de la PB con vacuna autógena en condiciones del estado de Guerrero.

\section{MATERIALES Y MÉTODOS}

Sitio de estudio. El presente estudio se realizó en el municipio de Cuajinicuilapa, Guerrero, México; en los ranchos El Cerrito, Las Lomas y El Charco Metepec, dedicados a la recría de ganado bovino comercial (Suizo Americano, Brahman y Beefmaster). El lugar se localiza geográficamente en el trópico sub-húmedo a $16^{\circ} 28^{\prime} 18^{\prime \prime}$ de Latitud Norte y $98^{\circ} 24^{\prime} 55^{\prime \prime}$ de Longitud Oeste, registrando temperaturas ambientales y humedad relativa promedio máximas durante el verano $\left(33.6^{\circ} \mathrm{C}\right.$ y $80 \%)$ y mínimas durante el invierno $\left(21.2^{\circ} \mathrm{C}\right.$ y $60 \%)(17)$.

Animales y tratamientos. En el experimento se usaron 18 hembras bovinas (Suizo Americano $x$ Brahman) púberes con edad (26.5 \pm 1.73 meses), peso $(364 \pm 1.73 \mathrm{~kg})$ y condición corporal (CC) (3.5 \pm 0.6 unidades) aptas para reproducir; las hembras fueron divididas en tres tratamientos de acuerdo al rancho ( 6 hembras/grupo). Tratamiento 1 (T1-inmunovacuna; El Charco Metepec), tratamiento 2 (T2-histovacuna; Las Lomas) y tratamiento 3 (T3-plasmaterapia; El Cerrito).

Muestreo y terapia. A 18 hembras bovinas se les tomó una muestra de tejido de sangre y piel; así como de diferente región anatómica (cuello y cola) con el fin de realizar una vacuna autógena para controlar la PB. Para ello, al T1inmunovacuna, se les colecto $20 \mathrm{~mL}$ de sangre de la vena yugular derecha. Por su parte, a las hembras del T2-histovacuna se les retiró quirúrgicamente los papilomas, posteriormente, fueron pesados, depositados y macerados con $480 \mathrm{~mL}$ de agua destilada en un mortero de porcelana $366 \mathrm{~g}$; al preparado se le añadió formol $(0.1 \mathrm{~mL} / 100 \mathrm{~mL})$, se dejó reposar por $24 \mathrm{~h}$ a temperatura de $24^{\circ} \mathrm{C}$, se decantó, filtró y se refrigeró a una temperatura de $8^{\circ} \mathrm{C}$. Por otra parte, a los animales del T3- plasmaterapia se les colectaron $10 \mathrm{~mL}$ de sangre de la vena coccígea, se centrifugó a 360 r.p.m. por 10 min y se recuperaron $4 \mathrm{~mL}$ de plasma, este fue depositado en frascos estériles a una temperatura de $8^{\circ} \mathrm{C}$. Posteriormente, fueron tratados los tres grupos de hembras bovinas; al T1 después de extraer la sangre fue aplicada vía subcutánea al lado izquierdo del cuello, al T2 y T3 $4 \mathrm{~mL}$ de la preparación fue administrado por la misma vía y lugar anatómico. A cada animal se le administró su propio preparado como vacuna autógena. Adicionalmente, durante el estudio fueron realizados 5 aplicaciones de la vacuna a cada hembra con intervalos entre aplicaciones de 7 d, antes de la administración se realizó el conteo y registro de los papilomas.

Manejo pre y experimental. Todos los animales se sometieron a un examen clínico exploratorio, donde se evaluó el estado físico, características y ubicación de los papilomas. Para seleccionar a los animales se tomó en cuenta el grado de infestación del hato, densidad de animales, edad, peso vivo y CC (Tabla 1 ). Se seleccionó la región del cuello como el área de muestreo, ya que era el lugar anatómico del animal con mayor número de papilomas.

Tabla 1. Características fisiológicas de los animales seleccionados para experimentación.

\begin{tabular}{ccccc}
\hline Tratamientos $^{\mathbf{1}}$ & $\mathbf{N}^{\mathbf{2}}$ & Edad $^{\mathbf{3}}$ & $\mathbf{P V}(\mathbf{k g})^{\mathbf{4}}$ & CC $^{\mathbf{5}}$ \\
\hline T1 & 6 & $26.3 \pm 1.6^{\mathrm{a}}$ & $366 \pm 58.7^{\mathrm{a}}$ & $3.3 \pm 0.5^{\mathrm{a}}$ \\
T2 & 6 & $27.0 \pm 1.1^{\mathrm{a}}$ & $377 \pm 66.2^{\mathrm{a}}$ & $3.7 \pm 0.8^{\mathrm{a}}$ \\
T3 & 6 & $26.2 \pm 2.5^{\mathrm{a}}$ & $349 \pm 76^{\mathrm{a}}$ & $3.5 \pm 0.5^{\mathrm{a}}$ \\
\hline
\end{tabular}

${ }^{1}$ Tratamiento 1, 2 y 3: T1, T2 y T3; ${ }^{2}$ Animales: $n$; ${ }^{3}$ Edad en meses; ${ }^{4}$ Peso vivo en kilogramos: PV (kg); ${ }^{5}$ Escala de condición corporal: CC - (1 al 5); Literal a entre variables denota que no hubo diferencias significativas $(p<0.05)$.

Alojamiento y alimentación. Las hembras bovinas experimentales fueron alojadas en potreros con el resto del hato de ganado bovino. El sistema de producción es semiintensivo, pastoreo diurno y encierro nocturno, la alimentación del ganado se basa en zacate Guinea (Panicum maximum) y Estrella africana (Cynodon nlemfuensis), con complementación alimenticia con rastrojo de maíz y sales minerales durante la época de secas.

Análisis estadísticos. Los datos generados del estudio fueron analizados bajo un diseño completamente al azar. Se realizó una estructura de covarianza autoregresivo, donde se midió el 
efecto del tratamiento, el periodo y la interacción del tratamiento $x$ periodo; todo esto con el procedimiento PROC MIXED, SAS (18). LoS resultados se presentan en promedio \pm e.e.m. y porcentajes.

Nota ética. Los procedimientos experimentales usadas en el estudio se ajustaron a las normas para el uso ético, cuidado y bienestar de los animales en investigación a nivel nacional e internacional $(19,20)$.

\section{RESULTADOS}

Al inicio del estudio se encontró una alta incidencia de papilomas en las hembras bovinas independientemente del tratamiento [( $\mathrm{T} 1$ (190 papilomas), T2 (95 papilomas) y T3 (100 papilomas) (Tabla 2)]. La aplicación de la vacuna autógena disminuyó significativamente (T1 y T3) el número de papilomas a través de las aplicaciones (1 (147 y 66 papilomas) a la 5 (10 y 9 papilomas); $(p<0.05)$ (Figura 1$)$. También existió una diferencia significativa (T1, T2 y T3) de acuerdo al periodo de aplicación de la vacuna (3 a la 5) disminuyó el número de papilomas (efecto periodo; $\mathrm{p}<0.05$ ) (Figura 2). Por su parte, se observó una disminución del número de papilomas en la cuarta y quinta aplicación en el T1 y T3 (interacción tratamiento x periodo; $\mathrm{p}<0.05$ ) (Figura 3). Finalmente, en la Tabla 2 se presenta la información en porcentajes sobre la disminución de los papilomas a través de la aplicación de la vacuna autógena.

Tabla 2. Disminución de la papilomatosis de acuerdo con la aplicación repetida de vacuna autógena en hembras bovinas.

\begin{tabular}{|c|c|c|c|c|c|c|c|}
\hline \multirow{2}{*}{ ID } & \multirow{2}{*}{ Rancho } & \multirow{2}{*}{ Papilomas } & \multicolumn{5}{|c|}{ Semanas } \\
\hline & & & 1 & 2 & 3 & 4 & 5 \\
\hline 1203419433 & $\mathrm{CM}$ & 35 & $20(57 \%)$ & $12(34 \%)$ & $7(20 \%)$ & - & - \\
\hline 1203419434 & $\mathrm{CM}$ & 8 & $7(88 \%)$ & $3(38 \%)$ & - & - & - \\
\hline 1203419435 & $\mathrm{CM}$ & 86 & $76(88 \%)$ & $51(59 \%)$ & $36(42 \%)$ & $13(15 \%)$ & $8(9 \%)$ \\
\hline 1203419436 & $\mathrm{CM}$ & 4 & $5(125 \%)$ & $5(125 \%)$ & $2(50 \%)$ & $1(25 \%)$ & - \\
\hline 1203419437 & $\mathrm{CM}$ & 21 & $8(38 \%)$ & $3(14 \%)$ & - & - & - \\
\hline 1203419438 & $\mathrm{CM}$ & 36 & $31(86 \%)$ & $13(36 \%)$ & $5(14 \%)$ & $3(8 \%)$ & $2(6 \%)$ \\
\hline 1203419394 & LM & 2 & - & - & - & - & - \\
\hline 1203419395 & LM & 6 & $5(14 \%)$ & $3(8 \%)$ & $3(8 \%)$ & $2(6 \%)$ & $2(6 \%)$ \\
\hline 1203419396 & LM & 19 & $16(84 \%)$ & $12(63 \%)$ & $12(63 \%)$ & $10(53 \%)$ & $8(42 \%)$ \\
\hline 1203419397 & LM & 26 & $21(81 \%)$ & $18(69 \%)$ & $18(69 \%)$ & $15(58 \%)$ & $12(46 \%)$ \\
\hline 1203419398 & LM & 39 & $35(90 \%)$ & $34(87 \%)$ & $34(87 \%)$ & $31(79 \%)$ & $32(82 \%)$ \\
\hline 1203419399 & LM & 3 & - & - & - & - & - \\
\hline 1203419440 & EC & 15 & $10(67 \%)$ & $7(47 \%)$ & $5(33 \%)$ & $1(7 \%)$ & - \\
\hline 1203419441 & $\mathrm{EC}$ & 4 & $3(75 \%)$ & - & - & - & - \\
\hline 1203419442 & EC & 2 & - & - & - & - & - \\
\hline 1203419443 & $\mathrm{EC}$ & 18 & $11(61 \%)$ & $6(33 \%)$ & $4(22 \%)$ & - & - \\
\hline 1203419444 & $\mathrm{EC}$ & 58 & $42(72 \%)$ & $34(59 \%)$ & $25(43 \%)$ & $17(29 \%)$ & $9(16 \%)$ \\
\hline 1203419445 & EC & 3 & - & - & - & - & - \\
\hline
\end{tabular}

Charco Metepec: CM, Las lomas: LM y El Cerrito: CE.- indica 0\%.

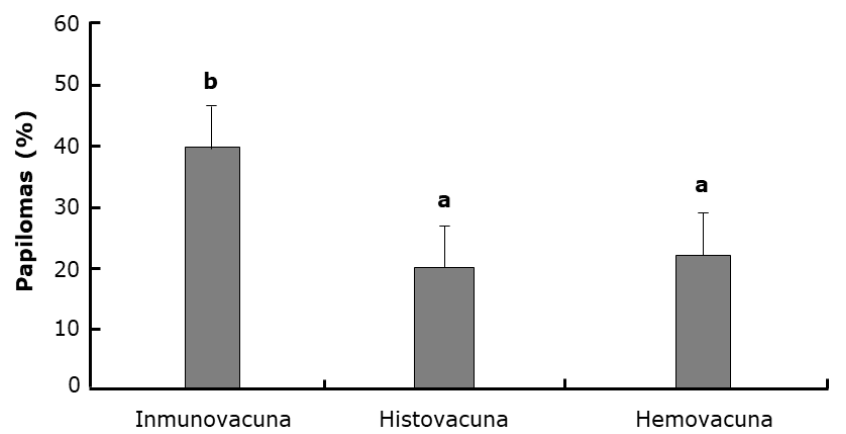

Figura 1. Porcentaje de papilomas en las hembras bovinas de los tres tratamientos de vacuna autógena (T1- inmunovacuna, T2histovacuna y T3- plasmaterapia). a,bMedias con distinta literal entre tratamientos difieren estadísticamente $(p<0.05)$.

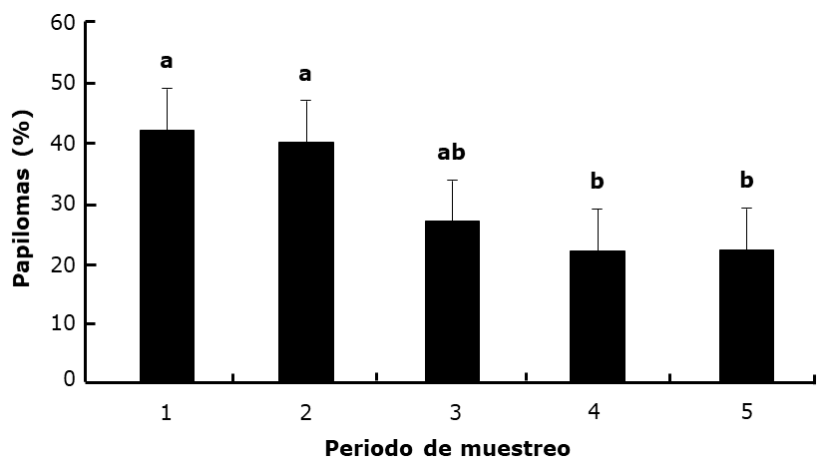

Figura 2. Efecto de la vacuna autógena en relación al tiempo de muestreo. a,bMedias con distinta literal entre tratamientos difieren estadísticamente $(p<0.05)$. 


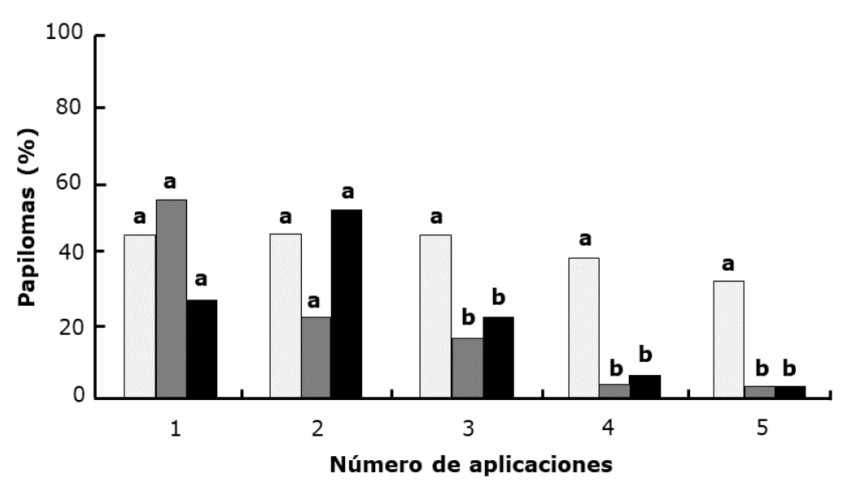

Figura 3. Efecto de la aplicación sobre el porcentaje de papilomas presentes después de la aplicación de vacuna autógena (T1, T2 y T3). a,bMedias con distinta literal entre tratamientos difieren estadísticamente $(p<0.05)$.

\section{DISCUSIÓN}

Los datos del presente estudio muestran que la aplicación repetida de vacuna autógena disminuyó significativamente el número de papilomas en las hembras bovinas en los T1 y T3 entre la cuarta y quinta aplicación. También muestran una interacción entre el tratamiento $x$ periodo en los T1 y T3. Previos estudios han descrito la epidemiologia de la papilomatosis y las diferentes especies que son susceptibles a la enfermedad viral (bóvidos, équidos, suinos, entre otros); sin embargo, las verrugas cutáneas son causadas por tipos virales específicos de especie $(4,6,12)$. En efecto, en los bovinos es más frecuente y fuerte la enfermedad de $\mathrm{PB}, \mathrm{y}$ cada región anatómica del animal tiene un tipo viral específico (VPB1 al 6) $(5,9)$. En el presente estudio la región anatómica seleccionada para muestrear fue la tabla del cuello, ya que era la más afectada por los papilomas, también se eligieron solo hembras bovinas debido a la alta densidad poblacional de hembras en el rancho de recría de ganado bovino. De hecho, Violet et al (21) evaluaron en su estudio el sexo, edad y ubicación de las lesiones causadas por las verrugas, donde encontraron mayor porcentaje en las hembras (77\%) que en los machos (43\%).

En el presente estudio se trabajó con 3 tratamientos en hembras bovinas jóvenes (26.2 22.5 meses); los cuales tuvieron una respuesta significativa al disminuir los papilomas en los T1 y T3. En este sentido, Aricapa et al. (13) trabajaron con hembras bovinas entre 12 y 20 meses de edad utilizando un tratamiento de autovacuna y los resultados que tuvieron fueron similares a los del presente trabajo. En contraste, Özyurtlu y Aslan (22) encontraron que la hemoterapia no es efectiva, pues las lesiones no llegan a desaparecer por completo. Al respecto, algunos estudios mencionan haber encontrado ADN viral a nivel de sangre, lo que significa que este método es un modo de transmisión de la PB, así como de otras enfermedades $(4,13)$. Por su parte, la plasmaterapia si puede ser utilizada con éxito ya que es específica, y con este tratamiento no existe riesgo de transmisión a otros animales de la misma especie (22).

Se ha reportado que la prevalencia de PB en los hatos de ganado bovino oscila en un $10 \%$ del ganado, aunque es muy variable por las características que presenta el virus y debido a diferentes factores de riesgo (propios del animal, edad, parásitos e inmunosupresores) $(23,24)$. Los datos del presente estudio coinciden con esta información ya que solamente el $10 \%$ de los animales de los tres hatos presentan la enfermedad viral. Contrariamente a estos resultados en el estado de Yucatán, México encontraron una prevalencia del $20 \%$ (25). Con lo anterior se podría pensar que no tiene repercusiones económicas importantes para los ganaderos, sin embargo, esto no es así ya que involucra otros factores que hacen que esto suceda.

De acuerdo con la literatura se reporta que la papilomatosis es la segunda neoplasia dermatológica en bovinos, después del carcinoma de las células escamosas (26). Lo anterior es debido a que la PB es de alta morbilidad, por ello se convierte en una enfermedad oncológica grave para los ganaderos porque es difícil evitar que afecte a otros animales del hato, convirtiéndose en un factor que ocasiona importantes pérdidas económicas en la ganadería bovina, debido al retraso en el desarrollo, baja condición corporal, reducción en la producción de leche, cojeras y pérdidas importantes por el descarte precoz de animales (25). Adicionalmente ocasiona en la ganadería perdidas de pieles por el mal aspecto ya que se dificulta su comercialización debido a que no pueden ser utilizadas en la industria de la peletería (3), así como el deterioro de la salud del animal debido a que la PB tiene tropismo por células de diferentes órganos importantes y vitales (papilomas esofágicos, carcinogénesis de la vejiga, entre otros) lo que podría causar la muerte del animal afectado por la enfermedad $(25,26,27,28,29)$.

En conjunto, los resultados del presente estudio se pueden resaltar que la efectividad del tratamiento en base a vacuna depende tanto de factores internos como externos del 
animal: los primeros a nivel inmunitario, edad, enfermedades secundarias, y los segundos involucran al clima, manejo, alimentación y la dosis utilizada para el tratamiento de los animales. Aunque la vacuna autógena se puede utilizar con buenos resultados, sería recomendable utilizarla individualmente para que no exista la posibilidad de contagios de esta y otras enfermedades que tienen que ver con fluidos corporales (30). Lo anterior, ya que en el presente estudio la vacuna se preparó por animal con tejido del propio bovino, para así evitar contagios si se administra a otros animales.

En conclusión, la administración repetida de la vacuna autógena en las hembras bovinas disminuyó el número de papilomas entre la cuarta y quinta administración de los tratamientos 1 y 3. Información alentadora para los productores de ganado bovino de regiones tropicales ya que evidencian alternativas sustentables para tratar a los animales infectados con PB.

\section{Conflicto de intereses}

Los autores del presente estudio declaramos que no existe conflicto de intereses con la publicación de este manuscrito.

\section{Agradecimientos}

Se agradece a los MVZ. Carlos Fabián Sala, Juan Orlando Rojas López y al M.C. Gabriel Mendoza Medel, por su apoyo técnico con los animales y procesamiento de las muestras en el laboratorio. Un agradecimiento especial a los dueños de los ranchos El Charco Metepec, Las Lomas y El Cerrito, por permitirnos trabajar con sus animales. Finalmente, se agradece al grupo de investigación "Sistemas de Producción Animal" de la ESMVZ-3/ UAGro por toda la colaboración brindada en el diseño del estudio y redacción del manuscrito.

\section{REFERENCIAS}

1. Orantes-Zebadúa MA, Platas-Rosado D, Córdova-Avalos V, De Los Santos-Lara M, Córdova-Avalos A. Caracterización de la ganadería de doble propósito en una región de Chiapas, México. Ecosist Rec Agrop. 2014; 1(1):49-58. https://era.ujat. $\mathrm{mx/}$ index.php/rera/issue/view/88

2. Espinosa-García JA, Góngora-González SF, García-Martínez A, Cervantes-Escoto FC, Mancilla-Rendón ME, Rangel-Quintos V. Estado del arte sobre la investigación e innovación tecnológica en ganadería bovina tropical. CONACYT: México; 2015. http://www.uco.es/zootecniaygestion/img/ pictorex/15 $13 \quad 27 \quad \mathrm{CL}$ 22.pdf

3. Jelínek R, Tachezy R. Cutaneous papillomatosis in cattle. J Comp Pathol. 2005; 132(1):70-81. https://doi.org/10.1016/j. jсpa.2004.07.001

4. Shafti-Keramat S, Schellenbacher C, Handisurya A, Christensen N, Reininger $B$, Brandt, $S$, et al. Bovine papillomavirus type 1 (BPV1) and BPV2 are closely related serotypes. Virology. 2009; 393(1):1-6. https://doi.org/10.1016/j. virol.2009.07.036
5. Maiolino $P$, Ozkul A, Sepici-Dincel $A$, Roperto F, Yücel G, Russo V, et al. Bovine papillomavirus type 2 infection and microscopic patterns of urothelial tumors of the urinary bladder in water buffaloes. Biomed Res Int. 2013; 2013:937918. http://dx.doi.org/10.1155/2013/937918

6. $\mathrm{Ng} \mathrm{T}$, Miller $\mathrm{M}$, Kondov $\mathrm{N}$, Dodd $\mathrm{E}$, Batac $F$, Manzer $M$, et al. Oral papillomatosis caused by Enhydra lutris papillomavirus 1 (EIPV-1) in southern sea otters (Enhydra lutris nereis) in California, USA. J Wildl Dis. 2015; 51(2):446-453. https://doi. org/10.7589/2014-06-152

7. Bravo I, Sanjosé S, Gottschling M. The clinical importance of understanding the evolution of papillomaviruses. Trends Microbiol. 2010; 18(10):432-438. https:// doi.org/10.1016/j.tim.2010.07.008

8. Lucena R, Rissi D, Kommers G, Pierezan F, Oliveira-Filho J, Macêdo J, et al. A retrospective study of 586 tumours in Brazilian cattle. J Comp Pathol. 2011; 145(1):20-24. https://doi.org/10.1016/j. jсра.2010.11.002 
9. Borzacchiello G. Roperto F. Bovine papillomaviruses, papillomas and cancer in cattle. Vet Res. 2008; 39(5):45. https://doi. org/10.1051/vetres:2008022

10. Buck C, Day $P$, Trus B. The papillomavirus major capsid protein L1. Virology. 2013; 445(1-2):169-174. https://doi. org/10.1016/j.virol.2013.05.038

11. Borzacchiello G. Bovine papillomavirus infections in animals. Commun Curr Res Educ Top Trends Appl Microbiol. 2007: 673-679. https://doi.org/10.1590/16784685-GMB-2016-0128.

12. Linsey $\mathrm{CJ}$, Almeida $\mathrm{ME}$, Vicari $\mathrm{CF}$, Carvalho A, Yaguiu A, Freitas AC, et al. Bovine papillomarivus DNA in milk, blood, urine, semen and spermatozoa of bovine papillomavirus-infected animals. Genet Mol Res. 2009; 8(1):310-318. https://doi. org/10.4238/vol8-1gmr573.

13. Aricapa $\mathrm{HJ}$, Dussan C, Marique H. Evaluación de la eficiencia de una vacuna contra la papilomatosis bovina frente a tres tratamientos diferentes. Rev Vet Zootec Cald. 2003; 12(2):48-55. http://vetzootec. ucaldas.edu.co/

14. Avki $S$, Turutoglu $H$, Simsek A, Unsal A. Clinical and immunological effects of Newcastle disease virus vaccine on bovine papillomatosis. Veterin Immunol Immunopathol. 2004; 98(1-2):9-16. https:// doi.org/10.1016/j.vetimm.2003.10.003.

15. Hemmatzadeh F, Fatemi A, Amini F. Therapeutic effects of fig tree latex on bovine papillomatosis. J Veterin Med. Infect Diseas Veterin Pub Healt. 2003; 50(10):473-476. https://doi.org/10.1046/ j.1439-0450.2003.00702.x

16. Börkü MK, Atalay O, Kibar M, Cam Y, Atasever $A$. Ivermectin is an effective treatment for bovine cutaneous papillomatosis. Res Veterin Sci. 2007; 83(3):360-363. https:// doi.org/10.1016/j.rvsc.2007.01.016.

17. García E. Modificaciones al sistema de clasificación climática de Köeppen. 2da ed. Instituto de Geografía, Universidad Nacional Autónoma de México: México, D.F; 1973. http://www.publicaciones.igg.unam.mx/ index.php/ig/catalog/book/83.
18. SAS Institute, SAS/STAT. 2004. User's guide statistics released 9.1 (2nd Ed.) SAS Institute, Inc. Cary. in:https://support. sas.com/documentation/onlinedoc/91pdf/ sasdoc 91/share ug 7261.pdf

19. NAM. Guide for the care and use of laboratory animals. Co-produced by the National Academy of Medicine-Mexico and the Association for assessment and accreditation of laboratory animal care international. 1st. Edition. Harlan: Mexico; 2002. https://grants.nih.gov/grants/olaw/ guide-for-the-care-and-use-of-laboratoryanimals.pdf

20. FASS. Guide for the care and use of agricultural animals in agricultural research and teaching. 3rd edition. Federation Animal Science Society: Savoy, USA; 2010. https:// www.adsa.org/Publications/FASS-2010-AgGuide.

21. Violet LL, Montes VD, Cardona AJ. Frecuencia de papilomatosis en bovinos (Bos taurus) del departamento de Córdoba, Colombia. Rev Colomb Cienc Anim - RECIA. 2017; 9(Supl 2):294-300. https://doi.org/10.24188/ recia.v9.n2.2017.611

22. Özyurtlu N, Aslan S. Homeopati ve Veteriner Hekimliginde Kullanimi. Veteriner Hekimler Dernegi Dergisi. 2007; 78(1):39-42. https://doi.org/10.18805/ijar.5586

23. Campo M. Vaccination against papillomavirus in cattle. Clin Dermatol. 1997; 15(2):275283. https://doi.org/10.1016/S0738$\underline{081 \times(96) 00165-4}$

24. Rojas-Anaya E, Cantú-Covarrubias A, MoralesÁlvarez JF. Detection and phylogenetic analysis of bovine papillomavirus in cutaneous warts in cattle in Tamaulipas, Mexico. Can J Vet Res. 2017; 80(4):262268. https://www.ncbi.nlm.nih.gov/pmc/ articles/PMC5052877/pdf/cjvr 10 262.pdf

25. Catroxo M, Martins A, Petrella S, Souza F, Nastari B. Ultrastructural study of bovine papillomavirus during outbreaks in Brazil. Internat J Morphol. 2013; 3(2):777784. http://dx.doi.org/10.4067/S0717$\underline{95022013000200068}$ 
26. Reis $M$, Slaviero $M$, Lorenzett $M$, Cruz $R$, Guimarães L, Pavarini $S$, et al. Neoplasmas bovinos diagnosticados no Setor de Patologia Veterinária da UFRGS, Porto Alegre (20052014). Pesquisa Vet Bras. 2017; 37(2):105109. https://doi.org/10.1590/s0100$\underline{736 \times 2017000200002}$

27. Roperto S, Borzacchiello G, Esposito I, Riccardi M, Urraro C, Cermola M, et al. Productive infection of bovine papillomavirus type 2 in the placenta of pregnant cows affected with urinary bladder tumors. PLoS One. 2012; 7:e33569. https://doi. org/10.1371/journal.pone.0033569
28. Lunardi $M$, Alcântara $B$, Otonel R, Rodrigues W, Alfieri A, Alfieri A. Bovine papillomavirus type 13 DNA in equine sarcoids. J Clin Microbiol. 2013; 51(7):2167-2171. https:// doi.org/10.1128/JCM.00371-13.

29. Savini F, Mancini S, Gallina L, Donati G, Casá $G$, Peli $A$, et al. Bovine papillomatosis: First detection of bovine papilloma-virus types $6,7,8,10$ and 12 in Italian cattle herds. Rev Vet J. 2016; 210:82-84. https://doi. org/10.1016/j.tvjl.2016.02.003.

30. Foldvari M, Kumar P. Recent progress in the application of nanotechnology for prevention and treatment of human papillomavirus infection. Ther Deliv. 2012; 3(8):10051017. https://doi.org/10.4155/tde.12.78. 